Brief Original Report

\title{
Can air pollution negate the health benefits of cycling and walking?
}

\author{
Marko Tainio $^{\mathrm{a}, *}$, Audrey J. de Nazelle ${ }^{\mathrm{b}}$, Thomas Götschi ${ }^{\mathrm{c}}$, Sonja Kahlmeier ${ }^{\mathrm{c}}$, David Rojas-Rueda ${ }^{\mathrm{d}, \mathrm{e}, \mathrm{f}}$, \\ Mark J. Nieuwenhuijsen d,e,f, Thiago Hérick de Ság ${ }^{g}$, Paul Kelly ${ }^{\mathrm{h}}$, James Woodcock ${ }^{\mathrm{a}}$ \\ a UKCRC Centre for Diet and Activity Research, MRC Epidemiology Unit, University of Cambridge School of Clinical Medicine, Institute of Metabolic Science, Cambridge, UK \\ b Centre for Environmental Policy, Imperial College London, London, UK \\ c Physical Activity and Health Unit, Epidemiology, Biostatistics and Prevention Institute, University of Zurich, Zurich, Switzerland \\ d Center for Research in Environmental Epidemiology (CREAL), Barcelona, Spain \\ e Universitat Pompeu Fabra (UPF), Barcelona, Spain \\ ${ }^{\mathrm{f}}$ Centro de Investigación Biomédica en Red de Epidemiología y Salud Pública (CIBERESP), Madrid, Spain \\ g Centre for Epidemiological Research in Nutrition and Health, School of Public Health, University of São Paulo, São Paulo, Brazil \\ ${ }^{\mathrm{h}}$ Physical Activity for Health Research Centre (PAHRC), University of Edinburgh, UK
}

\section{A R T I C L E I N F O}

\section{Article history:}

Received 9 October 2015

Received in revised 28 January 2016

Accepted 1 February 2016

Available online 5 May 2016

\section{Keywords:}

Physical activity

Air pollution

Bicycling

Walking

Mortality

Health Impact Assessment

Risk-Benefit Assessment

\begin{abstract}
A B S T R A C T
Active travel (cycling, walking) is beneficial for the health due to increased physical activity (PA). However, active travel may increase the intake of air pollution, leading to negative health consequences. We examined the riskbenefit balance between active travel related PA and exposure to air pollution across a range of air pollution and PA scenarios.

The health effects of active travel and air pollution were estimated through changes in all-cause mortality for different levels of active travel and air pollution. Air pollution exposure was estimated through changes in background concentrations of fine particulate matter $\left(\mathrm{PM}_{2.5}\right)$, ranging from 5 to $200 \mu \mathrm{g} / \mathrm{m} 3$. For active travel exposure, we estimated cycling and walking from 0 up to $16 \mathrm{~h}$ per day, respectively. These refer to long-term average levels of active travel and $\mathrm{PM}_{2.5}$ exposure.

For the global average urban background $\mathrm{PM}_{2.5}$ concentration $(22 \mu \mathrm{g} / \mathrm{m} 3)$ benefits of PA by far outweigh risks from air pollution even under the most extreme levels of active travel. In areas with $\mathrm{PM}_{2.5}$ concentrations of $100 \mu \mathrm{g} / \mathrm{m} 3$, harms would exceed benefits after $1 \mathrm{~h} 30 \mathrm{~min}$ of cycling per day or more than $10 \mathrm{~h}$ of walking per day. If the counterfactual was driving, rather than staying at home, the benefits of PA would exceed harms from air pollution up to $3 \mathrm{~h} 30 \mathrm{~min}$ of cycling per day. The results were sensitive to dose-response function (DRF) assumptions for $\mathrm{PM}_{2.5}$ and PA.

PA benefits of active travel outweighed the harm caused by air pollution in all but the most extreme air pollution concentrations.
\end{abstract}

(c) 2016 The Authors. Published by Elsevier Inc. This is an open access article under the CC BY license

(http://creativecommons.org/licenses/by/4.0/).

\section{Introduction}

Several health impact modelling (HIM) studies have estimated the health benefits and risks of active travel (cycling, walking) in different geographical areas (Mueller et al., 2015; Doorley et al., 2015). In most of these studies, the health benefits due to physical activity (PA) from increased active travel are significantly larger than the health risks caused by increases in exposure to air pollution.

Most of the existing active travel HIM studies have been carried out in cities in high income countries with relatively low air pollution levels (Mueller et al., 2015; Doorley et al., 2015). This raises the question on the risk-benefit balance in highly polluted environments. Health risks of air pollution are usually thought to increase linearly with increased

\footnotetext{
* Corresponding author.

E-mail address: mkt27@medschl.cam.ac.uk (M. Tainio).
}

exposure for low to moderate levels of air pollution, whereas the benefits of PA increase curvy-linearly with increasing dose (Kelly et al., 2014; World Health Organization, 2014). Thus, at a certain level of background air pollution and of active travel, risks could outweigh benefits, which would directly imply that, from a public health perspective, active travel could not be always recommended.

In this study we compare the health risks of air pollution with the PA-related health benefits from active travel across a wide range of possible air pollution concentrations and active travel levels. We use two thresholds to compare PA benefits and air pollution risks (Fig. 1): At the "tipping point" an incremental increase in active travel will no longer lead to an increase in health benefits (i.e. max. benefits have been reached). Increasing active travel even more could lead to the "breakeven point", where risk from air pollution starts outweighing the benefits of PA (i.e. there are no longer net benefits, compared to not engaging in active travel). 


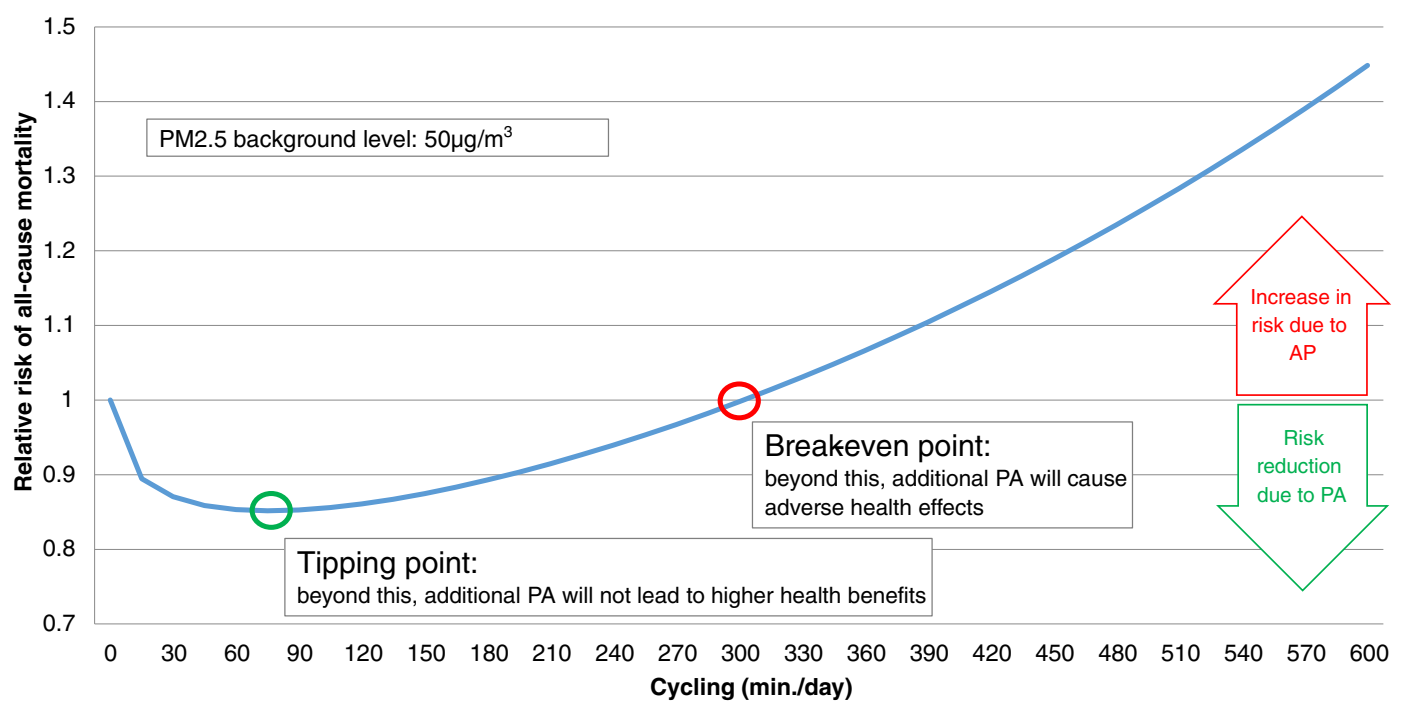

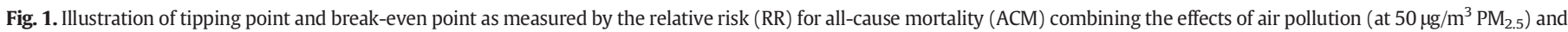
physical activity (cycling).

\section{Methods}

Our approach followed a general active travel HIM method (Mueller et al., 2015; Doorley et al., 2015). Air pollution exposures due to active travel were quantified by estimating the differences in the inhaled dose of fine particulate matter $\left(\mathrm{PM}_{2.5}\right)$ air pollution. We selected $\mathrm{PM}_{2.5}$ because it is a commonly used indicator of air pollution in active travel HIM studies (Mueller et al., 2015; Doorley et al., 2015), and because of the large health burden caused by $\mathrm{PM}_{2.5}$ (GBD 2013 Risk Factors Collaborators et al., 2015). For both air pollution and PA we used allcause mortality as the health outcome because there is strong evidence for its association with both long-term exposure to $\mathrm{PM}_{2.5}$ (Héroux et al., 2015) and long-term PA behaviour (Kelly et al., 2014).

The reduction in all-cause mortality from active travel was estimated by converting the time spent cycling or walking to metabolically equivalent of task (MET) and calculating the risk reduction using dose-response functions (DRFs) adapted from Kelly et al.'s ${ }^{3}$ metaanalysis. From the different DRFs reported in Kelly et al. (2014) we chose the one with the " 0.50 power transformation" as a compromise between linear and extremely non-linear DRFs. Non-linearity in a DRF means that the health benefits of increased active travel would level out sooner and a tipping point would be reached earlier than with more linear DRFs. See supplementary material for the sensitivity analysis with different DRFs. To convert cycling and walking time to PA we used the values of 4.0 METs for walking and 6.8 METs for cycling, based on the Compendium of Physical Activities (Ainsworth et al., 2011). The walking and cycling levels used in this study are assumed to reflect long-term average behaviour.

The health risks of $\mathrm{PM}_{2.5}$ were estimated by converting background $\mathrm{PM}_{2.5}$ concentrations to travel mode specific exposure concentrations, and by taking into account ventilation rate whilst being active. For background $\mathrm{PM}_{2.5}$ we used values between 5 and $200 \mu \mathrm{g} / \mathrm{m} 3$ with $5 \mu \mathrm{g} / \mathrm{m} 3$ intervals. We also estimated tipping points and break-even points for the average and most polluted cities in each region included in the World Health Organization (WHO) Ambient Air Pollution Database (World Health Organization (WHO), 2014), which contains measured and estimated background $\mathrm{PM}_{2.5}$ concentrations for 1622 cities around the world.

The mode specific exposure concentrations were estimated by multiplying background $\mathrm{PM}_{2.5}$ concentration by 2.0 for cycling or 1.1 for walking, based on a review of studies (Kahlmeier et al., 2014). The counterfactual scenario for the time spent cycling or walking was assumed to be staying at home (i.e. in background concentration of $\mathrm{PM}_{2.5}$ ). See supplementary file for the sensitivity analysis with counterfactual scenarios where cycling time would replace motorised transport time. The ventilation rates differences whilst at sleep, rest, cycling and walking were taken into account when converting exposure to inhaled dose. For sleep, rest, walking and cycling we used ventilation rates of 0.27 , $0.61,1.37$ and 2.55, respectively (de Nazelle et al., 2009; Johnson, 2002). The sleep time was assumed to be $8 \mathrm{~h}$ in all scenarios and the resting time was $16 \mathrm{~h}$ minus the time for active travel.

For the $\mathrm{PM}_{2.5}$ DRF we used a relative risk (RR) value of 1.07 per $10 \mu \mathrm{g} / \mathrm{m} 3$ change in exposure (World Health Organization, 2014). We assumed that DRF is linear from zero to maximum inhaled dose. As a sensitivity analysis we used non-linear integrated risk function from Burnett et al. (2014) (see supplementary material for details).

The model used for all calculations is provided in Lumina Decision Systems Analytica format in supplementary file 2 (readable with Analytica Free 101, http://www.lumina.com/products/free101/), and a simplified model containing the main results is provided in Microsoft Excel format in supplementary file 3.

\section{Results}

The tipping point and break-even point for different average cycling times and background $\mathrm{PM}_{2.5}$ concentrations are shown in Fig. 2. For half an hour of cycling every day, the background $\mathrm{PM}_{2.5}$ concentration would need to be $95 \mu \mathrm{g} / \mathrm{m} 3$ to reach the tipping point. In the WHO Ambient Air Pollution Database less than $1 \%$ of cities have $\mathrm{PM}_{2.5}$ annual concentrations above that level (World Health Organization (WHO), 2014). The break-even point for half an hour of cycling every day was at $160 \mu \mathrm{g} / \mathrm{m} 3$ (Fig. 2). For half an hour of walking the tipping point and break-even point appear at a background concentration level above $200 \mu \mathrm{g} / \mathrm{m} 3$ (Fig. S3, supplementary file). For the average urban background $\mathrm{PM}_{2.5}$ concentration $(22 \mu \mathrm{g} / \mathrm{m} 3)$ in the WHO database, the tipping point would only be reached after $7 \mathrm{~h}$ of cycling and $16 \mathrm{~h}$ of walking per day.

Tables S2 and S3 (supplementary file) show the tipping point for cycling and walking, respectively, in different regions of the world. In the most polluted city in the database (Delhi, India, background concentration of $153 \mu \mathrm{g} / \mathrm{m} 3$ ), the tipping and break-even points were 30 and 45 min of cycling per day, respectively (Table S2, supplementary file). In most global regions the tipping points for the most polluted cities $(44 \mu \mathrm{g} / \mathrm{m} 3$ to $153 \mu \mathrm{g} / \mathrm{m} 3)$ varied between 30 and 120 min per day for cycling, and 90 min to $6 \mathrm{~h} 15 \mathrm{~min}$ per day for walking (Table S3, supplementary material). 


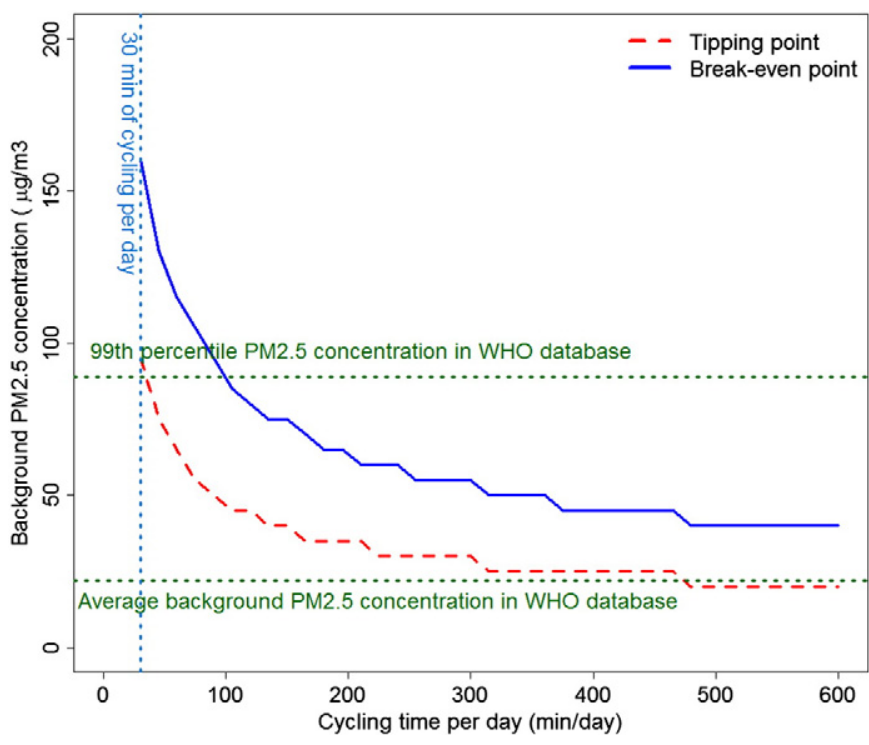

Fig. 2. Tipping and break-even points for different levels of cycling (red dashed line and blue solid line, respectively) (minutes per day, x-axis) and for different background $\mathrm{PM}_{25}$ concentrations (y-axis). Green lines represent the average and 99th percentile background $\mathrm{PM}_{2.5}$ concentrations in World Health Organization (WHO) Ambient Air Pollution Database (World Health Organization (WHO), 2014).

When we assumed that time spend cycling would replace time driving a car, benefits always exceeded the risks in the background air pollution concentrations below $80 \mu \mathrm{g} / \mathrm{m} 3$, a concentration exceeded in only $2 \%$ of cities (World Health Organization (WHO), 2014). Other sensitivity analyses showed that the results are sensitive to the shape of the DRF functions. With the linear DRF for active travel the break-even point would be reached with background $\mathrm{PM}_{2.5}$ concentrations of $170 \mu \mathrm{g} / \mathrm{m} 3$ regardless of the active travel time (Fig. S4, supplementary material); a level not currently found in any of the cities in the WHO air pollution database (World Health Organization (WHO), 2014). With the most curved DRF ( 0.25 power) the $\mathrm{PM}_{2.5}$ concentration where harms exceed benefits for $1 \mathrm{~h}$ of cycling per day would drop from $150 \mu \mathrm{g} / \mathrm{m} 3$ to $130 \mu \mathrm{g} /$ $\mathrm{m} 3$ (Fig. S4, supplementary material), a level currently found only in 9 cities (World Health Organization (WHO), 2014). With a non-linear $\mathrm{DRF}$ for $\mathrm{PM}_{2.5}$ the break-even point was not reached in any background $\mathrm{PM}_{2.5}$ concentration when using "power 0.50" DRF for cycling and walking. Other input value modifications had small or insignificant impact to the results.

\section{Discussions}

This study indicates that, practically, air pollution risks will not negate the health benefits of active travel in urban areas in the vast majority of settings worldwide. Even in areas with high background $\mathrm{PM}_{2.5}$ concentrations, such as $100 \mu \mathrm{g} / \mathrm{m} 3$, up to $1 \mathrm{~h} 15 \mathrm{~min}$ of cycling and $10 \mathrm{~h} 30 \mathrm{~min}$ of walking per day will lead to net reduction in all-cause mortality (Fig. S5, supplementary material). This result is supported by epidemiological studies that have found the statistically significant protective effects of PA even in high air pollution environments (Matthews et al., 2007; Andersen et al., 2015). However, a small minority engaging in unusually high levels of active travel (i.e. bike messengers) in extremely polluted environments may be exposed to air pollution such that it negates the benefits of PA.

Some considerations of the limitations and the strengths of our study need to be applied when generalising these findings.

In this analysis we took into account only the long-term health consequences of regular PA and chronic exposure to $\mathrm{PM}_{2.5}$. Impacts of short-term air pollution episodes, where concentrations significantly exceed the average air pollution levels for a few days, may induce additional short term health effects. We have also only worked with all- cause mortality and have, thus, not taken into account the morbidity impact.

For the health risks of air pollution we only estimated the increased risk during cycling and walking, not the overall health risk from everyday air pollution. Air pollution causes a large burden of diseases all over the world (Burnett et al., 2014) and reducing air pollution levels would provide additional health benefits. Since transport is an important source of air pollution in urban areas, mode shifts from motorised transport to active travel would not only improve health in active travellers, but also help to reduce air pollution exposures for the whole population (Johan de Hartog et al., 2010).

The results are sensitive to assumptions of the linearity of dose-response relationships between active travel-related PA and health benefits, and between $\mathrm{PM}_{2.5}$ and adverse health effects. With linear DRFs for PA the benefits always exceeded the risks at all levels of $\mathrm{PM}_{2.5}$ concentrations. Evidence for a linear DRF for high $\mathrm{PM}_{2.5}$ concentrations is small and, for example, the Global Burden of Disease study applied non-linear, disease specific DRFs for $\mathrm{PM}_{2.5}$ (Burnett et al., 2014). If the risks of $\mathrm{PM}_{2.5}$ level out after $\mathrm{PM}_{2.5}$ concentrations over $100 \mu \mathrm{g} / \mathrm{m} 3$, the health benefits of PA would always exceed the risks of $\mathrm{PM}_{2.5}$.

It should also be taken into account that the results are based on generally representative values without detailed information on local conditions, or from the background PA and disease history of individuals. For individuals highly active in non-transport domains the benefits from active travel will be smaller, and vice versa.

\section{Conclusions}

The benefits from active travel generally outweigh health risks from air pollution and therefore should be further encouraged. When weighing long-term health benefits from PA against possible risks from increased exposure to air pollution, our calculations show that promoting cycling and walking is justified in the vast majority of settings, and only in a small number of cities with the highest $\mathrm{PM}_{2.5}$ concentration in the world cycling could lead to increase in risk.

\section{Author contributions}

MT made the calculations and drafted the first version of the manuscript. AJN, TG, MJN, SK, THS, DRR, PK and JW participated in designing the scope of the study. AJN and TG helped to clarify the message of the study. All authors contributed to the writing of this paper. All authors approved the final version to be submitted for consideration of publication.

Conflict of interest statement

The authors declare that there are no conflicts of interests.

\section{Transparency document}

The Transparency document associated with this article can be found, in the online version.

\section{Acknowledgments}

MT and JW: The work was undertaken by the Centre for Diet and Activity Research (CEDAR), a UKCRC Public Health Research Centre of Excellence. Funding from the British Heart Foundation, Cancer Research UK, Economic and Social Research Council, Medical Research Council, the National Institute for Health Research, and the Wellcome Trust, under the auspices of the UK Clinical Research Collaboration, is gratefully acknowledged.

AJN, DRR, MJN, SK and TG: The work was supported by the project Physical Activity through Sustainable Transportation Approaches (PASTAs) funded by the European Union's Seventh Framework Program under EC-GA no. 602624-2 (FP7-HEALTH-2013-INNOVATION-1). The 
sponsors had no role in the study design; in the collection, analysis, and interpretation of data; in the writing of the report; and in the decision to submit the article for publication.

JW is supported by an MRC Population Health Scientist fellowship. THS is supported by the Brazilian Science without Borders Scheme (process number: 200358/2014-6) and the Sao Paulo Research Foundation (process number: 2012/08565-4).

\section{References}

Ainsworth, B.E., Haskell, W.L., Herrmann, S.D., et al., 2011. 2011 compendium of physical activities: a second update of codes and MET values. Med. Sci. Sports Exerc. 43 (8), 1575-1581. http://dx.doi.org/10.1249/MSS.0b013e31821ece12.

Andersen, Z.J., de Nazelle, A., Mendez, M.A., et al., 2015. A study of the combined effects of physical activity and air pollution on mortality in elderly urban residents: the Danish diet, cancer, and health cohort. Environ. Health Perspect. 123 (6), 557-563. http://dx. doi.org/10.1289/ehp.1408698.

Burnett, R.T., Pope, C.A., Ezzati, M., et al., 2014. An integrated risk function for estimating the global burden of disease attributable to ambient fine particulate matter exposure. Environ. Health Perspect. 122 (4), 397-403. http://dx.doi.org/10.1289/ehp.1307049.

de Nazelle, A., Rodríguez, D.A., Crawford-Brown, D., 2009. The built environment and health: impacts of pedestrian-friendly designs on air pollution exposure. Sci. Total Environ. 407 (8), 2525-2535. http://dx.doi.org/10.1016/j.scitotenv.2009.01.006.

Doorley, R., Pakrashi, V., Ghosh, B., July 2015. Quantifying the health impacts of active travel: assessment of methodologies. Transp. Rev. 2015, 1-24. http://dx.doi.org/10. 1080/01441647.2015.1037378.

GBD 2013 Risk Factors CollaboratorsMH, Forouzanfar, Alexander, L., et al., 2015. Global, regional, and national comparative risk assessment of 79 behavioural, environmental and occupational, and metabolic risks or clusters of risks in 188 countries, 19902013: a systematic analysis for the Global Burden of Disease Study 2013. Lancet (London, England) 6736 (15), 1990-2013. http://dx.doi.org/10.1016/S01406736(15)00128-2.
Héroux, M.-E., Anderson, H.R., Atkinson, R., et al., 2015. Quantifying the health impacts of ambient air pollutants: recommendations of a WHO/Europe project. Int. J. Public Health 619-627 http://dx.doi.org/10.1007/s00038-015-0690-y.

Johan de Hartog, J., Boogaard, H., Nijland, H., Hoek, G., 2010. Do the health benefits of cycling outweigh the risks? Environ. Health Perspect. 118 (8), 1109-1116. http://dx. doi.org/10.1289/ehp.0901747.

Johnson, T., 2002. A guide to selected algorithms, distribution, and databases used in exposure models developed by the office of air quality planning and standards. U.S. Environmental Protection Agency. Available at: http://www2.epa.gov/sites/ production/files/2013-08/documents/report052202.pdf.

Kahlmeier, S., Schweizer, C., Rojas-Rueda, D., Nieuwenhuijsen, M., Nazelle, A. de, Bode, O. 2014. Development of the Health Economic Assessment Tools (HEAT) for Walking and Cycling - Meeting Background Document. Bonn, Germany.

Kelly, P., Kahlmeier, S., Götschi, T., et al., 2014. Systematic review and meta-analysis of reduction in all-cause mortality from walking and cycling and shape of dose response relationship. Int. J. Behav. Nutr. Phys. Act. 11 (1), 132. http://dx.doi.org/10.1186/ s12966-014-0132-X.

Matthews, C.E., Jurj, A.L., Shu, X.O., et al., 2007. Influence of exercise, walking, cycling, and overall nonexercise physical activity on mortality in Chinese women. Am. J. Epidemiol. 165 (12), 1343-1350. http://dx.doi.org/10.1093/aje/kwm088.

Mueller, N., Rojas-Rueda, D., Cole-Hunter, T., et al., 2015. Health impact assessment of active transportation: A systematic review. Prev. Med. (Baltim) http://dx.doi.org/10. 1016/j.ypmed.2015.04.010.

World Health Organization (WHO), 2014. Ambient air pollution database. Available at: http://apps.who.int/gho/data/view.main.AMBIENTCITY2014?lang=en Accessed August 10, 2015.

World Health Organization, 2014. WHO expert meeting: methods and tools for assessing the health risks of air pollution at local, national and international level. Meeting report Copenhagen, Denmark. Available at: http://www euro.who.int/ data/assets/ pdf_file/0010/263629/WHO-Expert-Meeting-Methods-and-tools-for-assessing-thehealth-risks-of-air-pollution-at-local,-national-and-international-level.pdf. 